\title{
Telomerase gets Wnt talking
}

What do Wnt- $\beta$-catenin signalling and telomerase have in common? Both can activate quiescent epidermal stem cells in vivo. However, how telomerase does this, and whether its role in this process is linked to Wnt- $\beta$-catenin signalling, was unknown. Steven Artandi and colleagues now reveal that the telomerase reverse transcriptase (TERT) portion of telomerase interacts with the ATP-dependent chromatin remodelling factor BRG1 (also ...TERT activates Wnt signalling in vivo...

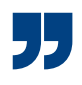
known as SMARCA4), at specific chromatin sites in Wnt-dependent target genes, to regulate Wnt$\beta$-catenin-mediated transcription.

$\beta$-Catenin is stabilized in response to Wnt ligands, which allows it to act as a transcriptional co-activator at Wnt promoters. Telomerase consists of several molecules, including TERT, telomerase RNA, dyskerin and telomerase Cajal body protein 1 , and is well known for its ability to add DNA repeats to chromosome ends. As the TERT component of telomerase activates stem cells, the authors purified TERT protein complexes from HeLa cells in an attempt to understand telomerasemediated signalling. BRG1 was identified in the TERT complexes, and also shown to co-precipitate with TERT from mouse embryonic stem (ES) cell samples. As BRG1 is known to interact with $\beta$-catenin, the authors investigated the effects of BRG1 and TERT on Wnt transcription. TERT increases Wnt-regulated promoter activity when $\beta$-catenin is stabilized in cells. As it is unable to activate Wnt promoter activity in the absence of BRG1, TERT seems to work together with BRG1 and $\beta$-catenin to activate Wnt target gene transcription.

The role of TERT in Wnt- $\beta$ catenin signalling was next assessed in vivo. Wnt signalling in the gastrointestinal tract - a stem cell niche - is increased when TERT is overexpressed in mice. By contrast, the ability of the Wnt ligand WNT3A to induce Wnt target gene expression is strongly reduced when TERT is deleted in mouse ES cells. These data suggest that TERT activates Wnt signalling in vivo and in ES cells, but can it alter the biological outcome of Wnt signalling?

Wnt- $\beta$-catenin signalling is involved in anterior-posterior axis formation in Xenopus laevis. Depletion of TERT in X. laevis embryos using morpholinos leads to a defect in anterior-posterior axis formation, which resembles the phenotype of mouse embryos that are deficient in Wnt signalling. TERT depletion also compromises $X$. laevis posterior development, which is consistent with the role of WNT3A in vertebrate posterior development. WNT3A partly regulates posterior development by regulating the homeobox gene $C d x 1$. Knockdown of TERT in mouse ES cells suppresses WNT3A-mediated $C d x 1$ induction, and depletion of TERT in X. laevis reduces the level of $c d x 1, c d x 2$ and $c d x 3$ expression. These data highlight the importance of TERT in Wnt$\beta$-catenin signalling in ES cells and $X$. laevis development.

Finally, how does TERT regulate the transcription of Wnt- $\beta$-catenin target genes? Chromatin immunoprecipitation reveals that TERT, together with $\beta$-catenin and BRG1, binds DNA fragments that contain elements involved in Wnt- $\beta$-catenin transcription in cultured cells and in the mouse gastrointestinal tract. This study provides new information on how Wnt- $\beta$-catenin signalling initiates gene transcription, and provides mechanistic insight into how telomerase functions in signalling processes.

Katharine H. Wrighton

ORIGINAL RESEARCH PAPER Park, J.-l. et al. Telomerase modulates Wnt signalling by association with target gene chromatin. Nature 460, 66-72 (2009)

FURTHER READING Angers, S. \& Moon, R. T. Proximal events in Wnt signal transduction. Nature Rev. Mol. Cell Biol. 10, 468-477 (2009) 